\title{
Neue Richtlinien der SAMW zu «Palliative Care»
}

Für die SAMW ist die Bedeutung der Palliative Care in der Medizin unbestritten; auf Initiative ihrer Zentralen Ethikkommission (ZEK) unterstützt sie deshalb schon seit einiger Zeit Projekte im Bereich der Forschung und Lehre in diesem Gebiet. Die ZEK hat zudem in mehreren der kürzlich ausgearbeiteten und veröffentlichten medizinisch-ethischen Richtlinien mit Nachdruck auf die zentrale Rolle von Palliative Care hingewiesen. Dabei hat sie festgestellt, dass ein Bedürfnis nach spezifischen Richtlinien für diesen Bereich besteht. Diese hätten die Aufgabe, Palliative Care zu definieren und Konzepte, den Platz im therapeutischen Behandlungskonzept, den Anwendungsbereich sowie das Verhältnis zur kurativen Medizin zu klären.

«Empathie» kann als Grundlage von Palliative Care bezeichnet werden. Diese Art von Empathie ist in erster Linie Gegenstand der Erziehung, muss aber im Laufe der Ausbildung vertieft und verfeinert, durch ethische Reflexion gestärkt, von einem humanistischen Verständnis der Beziehung von Patient und medizinischem Personal getragen und während der Fort- und Weiterbildung immer wieder aufgefrischt werden - und dies unabhängig von der jeweiligen Fachdisziplin der Ärzte, der diversen Therapeuten und der anderen Mitglieder des Pflegeteams.

Ein weiterer starker Akzent dieser Richtlinien liegt auf der wechselseitigen Anerkennung der Ärzte und ihrer Partner im Bereich der Pallia- tive Care. Es braucht die enge Zusammenarbeit bei der Festlegung des Behandlungsplans. Dieser soll im Konsens, gemeinsam mit dem Patienten und seinen Angehörigen, erarbeitet und regelmässig dem Krankheitsverlauf angepasst werden.

In diesem Zusammenhang ist die Bedeutung einer offenen und ehrlichen Information und Kommunikation zu unterstreichen. Kurative Behandlung und Palliative Care dürfen nicht unabhängig voneinander zum Einsatz kommen, sondern sollen ein harmonisches Ganzes bilden. Die sogenannte symptomatische Behandlung ist sowohl zum Bereich der kurativen Medizin als auch zur Palliative Care zu zählen. Je mehr die kurative Behandlung an ihre Grenzen stösst, gewinnt die Palliative Care an Bedeutung; dabei steht immer weniger die Krankheit, sondern mehr und mehr der Patient und seine Lebensqualität im Vordergrund. Selbstverständlich muss Palliative Care durch Fallanalysen und Forschungsprojekte ihre Methoden evaluieren, um so ihre Wirksamkeit zu erhöhen.

Wir laden nicht nur die Ärztinnen und Ärzte, sondern auch alle anderen Partner in der Pflege und aus dem Bereich Palliative Care ein, den Richtlinienentwurf auf unserer Website (www.samw.ch) zur Kenntnis zu nehmen und dazu Stellung zu nehmen.

Prof. Michel B. Vallotton, Präsident ZEK

Prof. Peter Suter, Präsident SAMW 